\title{
EFEITO DO COZIMENTO CONVENCIONAL SOBRE OS CAROTENÓIDES PRÓ-VITAMÍNICOS "A" DA POLPA DO PIQUI (Caryocar brasiliense Camb)
}

\author{
MARIA ISABEL LIMA RAMOS * \\ MARINA CASTILHOS SOUZA UMAKI ** \\ PRISCILA AIKO HIANE * \\ MANOEL MENDES RAMOS FILHO *
}

\begin{abstract}
Visou-se caracterizar os carotenóides, avaliar sua atividade pró-vitamínica "A" e verificar as perdas destes pigmentos decorrentes do cozimento convencional da polpa do piqui (Caryocar brasiliense Camb) com arroz, simulando o preparo de acordo com os hábitos regionais. Tanto as amostras cruas quanto as cozidas apresentaram os mesmos carotenóides: $\beta$-caroteno, $\zeta$-caroteno, criptoflavina, $\quad \beta$-criptoxantina, anteraxantina, zeaxantina e mutatoxantina. $O$ valor médio de carotenóides foi de 231,09 e $154,06 \mu \mathrm{g} / \mathrm{g}$ de polpa crua e cozida, respectivamente. O teor de vitamina "A" correspondeu a $494 \mathrm{ER} / 100 \mathrm{~g}$ de polpa in natura e $375 \mathrm{ER} / 100 \mathrm{~g}$ de polpa após o cozimento. $\mathrm{Em}$ todas as amostras analisadas, $o \beta$-caroteno foi o principal pigmento responsável pela atividade próvitamínica "A" encontrada. A perda de carotenóides decorrente do cozimento convencional da polpa do piqui ( $C$. brasiliense $\mathrm{Camb}$ ) foi em média de $30,25 \%$, correspondendo à perda média de $12,11 \%$ no teor de vitamina "A".
\end{abstract}

\section{INTRODUÇÃO}

O piqui, pequi ou pequiá é um fruto drupáceo, globoso, contendo de uma a quatro sementes volumosas, protegidas por endocarpo lenhoso, eriçado de espinhos delgados e agudos, com uma amêndoa grande e carnosa. Floresce de novembro a dezembro e frutifica de janeiro a abril. O piqui da espécie Caryocar brasiliense Camb aparece na região Centro-Oeste do Brasil, na qual predomina o cerrado. Outras espécies deste fruto estão distribuídas desde a região da Amazônia até a Bahia (8).

* Professores, Departamento de Tecnologia de Alimentos e Saúde Pública (DTA), Universidade Federal de Mato Grosso do Sul, Campo Grande, MS. (e-mail: mmramosf@nin.ufms.br).

** Bolsista do CNPq, Acadêmica do Curso de Farmácia da UFMS. 
A massa que recobre as sementes dos frutos é amarela, pastosa, farinácea e oleaginosa, fornece óleo comestível e é utilizada como condimento no preparo de arroz, carne, frango e guariroba, conferindo-Ihes sabor especial. As amêndoas contidas na semente fornecem óleo que pode ser utilizado para os mais variados fins $(8,18,2)$.

O aspecto mais significativo dos carotenóides, além da cor que proporcionam aos alimentos, é o fato de representarem a principal fonte de vitamina "A" em países em desenvolvimento, nos quais sua deficiência é considerada como um dos mais graves problemas $(19,20)$.

Vários estudos, realizados em algumas regiões do Brasil, detectaram a prevalência de hipovitaminose A. Estima-se que os alimentos de origem vegetal, em especial as hortaliças verdes folhudas, possam servir como fonte alternativa e barata desta vitamina em alguns países em desenvolvimento, devido aos preços elevados dos alimentos de origem animal $(6,17)$.

Em geral, os valores de vitamina "A", disponíveis na literatura, são baseados nos teores totais de carotenóides, não levando em conta apenas os carotenóides com atividade pró-vitamínica "A". Assim sendo, estes valores podem ser superestimados, já que incluem carotenóides inativos e estereoisômeros de carotenóides $(17,19)$.

São consideradas como importantes propriedades físicas e químicas dos carotenóides: a inibição do oxigênio; a absorção de luz; o bloqueio dos radicais livres mediante reações; o seu caráter lipofílico, sua insolubilidade em água; sua facilidade de isomerização e oxidação; além sua capacidade de unir superfícies hidrofílicas (14).

Os carotenóides têm sido relacionados com a intensificação do sistema imunológico e a redução do risco de doenças degenerativas como o câncer, enfermidades cardiovasculares, degeneração macular, relacionada com a idade e formação de catarata. Tais efeitos biológicos são independentes da atividade da pró-vitamina "A" e têm sido atribuídos à propriedade antioxidante dos carotenóides, porém este mecanismo ainda não está bem esclarecido $(5,13,14)$.

Os carotenóides são susceptíveis a isomerização e oxidação durante o processamento e a estocagem, resultando em perda de cor e da atividade biológica, além da formação de compostos voláteis que podem conferir aromas e sabores desejáveis ou indesejáveis em alguns alimentos. A ocorrência de oxidação depende da presença de oxigênio, metais, enzimas, lipídios insaturados e antioxidantes; exposição a luz, tipo e estado físico do carotenóide presente; severidade do tratamento; material da embalagem e condições de estocagem. A alteração da composição dos carotenóides durante a preparação doméstica, processamento industrial e 
estocagem foi revisada recentemente (15). Devido à susceptibilidade destes pigmentos às reações de degradação torna-se importante avaliar as alterações causadas por processamentos térmicos diversos, o que tem despertado interesse de alguns pesquisadores $(4,6,11,17)$.

O objetivo deste estudo foi avaliar o efeito do cozimento convencional, de acordo com a culinária regional, sobre os carotenóides pró-vitamínicos " $\mathrm{A}$ " da polpa do piqui (Caryocar brasiliense Camb), considerando a utilização deste fruto no preparo de pratos típicos regionais, como o arroz com piqui.

\section{MATERIAL E MÉTODOS}

\subsection{MATERIAL}

\subsubsection{Preparo das amostras}

Os frutos maduros de piqui (Caryocar brasiliense Camb) foram obtidos no período de safra (janeiro a abril), junto ao Mercado Municipal de Campo Grande - MS. No laboratório, os caroços foram separados e selecionados conforme a maturação e a polpa removida mediante fatiamento. A polpa foi separada em lotes de aproximadamente 20 gramas para as análises dos carotenóides, tanto nas amostras cruas como nas cozidas, sendo que a polpa utilizada para o cozimento foi pesada anteriormente à adição da água e do arroz.

\subsubsection{Tratamento térmico}

Para o cozimento convencional, aqueceu-se a polpa de piqui fatiada com pequenas porções de água até cozimento completo (aproximadamente 10 minutos). Em seguida, adicionou-se $\mathrm{o}$ arroz e a água, mantendo-se aquecimento em fogo brando por cerca de 30 minutos, conforme recomendado pela culinária regional $(2,18)$.

\subsection{MÉTODOS}

\subsubsection{Determinação qualitativa de carotenóides}

O método para determinação dos carotenóides foi baseado no procedimento descrito por RODRIGUEZ e al. (16) e ALMEIDA e PENTEADO (1).

As amostras cruas e as cozidas, após o resfriamento, foram trituradas e homogeneizadas em multiprocessador doméstico e os pigmentos extraídos 
com acetona resfriada. $\mathrm{O}$ extrato acetônico de carotenóides foi transferido para éter de petróleo.

Em função do alto teor de lipídios nas amostras, o extrato etéreo de carotenóides foi submetido à reação de saponificação, utilizando-se solução metanólica de hidróxido de potássio a $30 \%(\mathrm{p} / \mathrm{v})$, no mesmo volume da solução de pigmentos em éter de petróleo. Esta mistura foi mantida em constante agitação durante $2 \mathrm{~h}$, com posterior repouso por $24 \mathrm{~h}$ em temperatura ambiente e ao abrigo da luz (19).

A separação dos pigmentos foi obtida por cromatografia em coluna aberta, empacotada com óxido de magnésio e celite (1:2), efetuando-se eluições com gradiente de acetona em éter de petróleo, que variaram de 1 a $25 \%$.

Para a identificação dos carotenóides considerou-se a ordem de eluição na coluna, o espectro de absorção na região visível e ultra-violeta, os valores das relações de frente (Rf) em cromatografia de camada delgada e reações químicas para verificação de grupamentos epóxidos e hidroxilas.

\subsubsection{Determinação quantitativa de carotenóides}

$\mathrm{Na}$ quantificação de cada pigmento foram utilizados os valores dos coeficientes de extinção $\left(E_{1 \mathrm{~cm}}{ }^{1 \%}\right)$, citados por DAVIES (7) e GODOY (9). O cálculo do valor de vitamina " $A$ " foi efetuado através da atividade próvitamínica "A" de cada carotenóide precursor, de acordo com BAUERNFEIND (3).

\section{RESULTADOS E DISCUSSÃo}

\subsection{IDENTIFICAÇÃO E QUANTIFICAÇÃO DOS PRINCIPAIS CAROTENÓIDES PRESENTES NA POLPA DO PIQUI (Caryocar brasiliense $\mathrm{Camb}$ )}

Dos pigmentos extraídos da polpa do piqui (Caryocar brasiliense Camb), tanto das amostras cruas como das cozidas, foram separados e identificados os carotenóides: $\beta$-caroteno, $\zeta$-caroteno, criptoflavina, $\beta$-criptoxantina, anteraxantina, zeaxantina e mutatoxantina. $O$ valor médio de carotenóides encontrado foi de 231,09 e 154,06 $\mu \mathrm{g} / \mathrm{g}$ de polpa crua e cozida, respectivamente (Tabela 1).

Entre os pigmentos da polpa crua do piqui, a anteraxantina foi encontrada em maior porcentagem $(40,54 \%)$, seguida pela zeaxantina $(34,24 \%)$, criptoflavina $(7,70 \%), \beta$ - caroteno $(6,35 \%), \beta$-criptoxantina $(5,25 \%), \zeta$ caroteno $(4,05 \%)$ e mutatoxantina $(1,87 \%)$. Após o cozimento convencional 
foram detectadas zeaxantina (51,75\%), anteraxantina $(20,30 \%)$, criptoflavina $(8,22 \%), \quad \beta$-caroteno $(7,90 \%), \quad \zeta$-caroteno $(5,30 \%), \quad \beta$ criptoxantina $(5,18 \%)$, e mutatoxantina $(1,35 \%)$.

Embora RODRIGUEZ-AMAYA (14) tenha incluído o piqui (Caryocar villosum) entre os frutos contendo altos teores de $\mathrm{Y}$-caroteno, o mesmo não foi detectado na polpa do piqui (Caryocar brasiliense Camb) utilizada nesta pesquisa.

\section{TABELA 1 - COMPOSIÇÃO QUANTITATIVA DE CAROTENÓIDES NA POLPA DO PIQUI (Caryocar brasiliense Camb), EXPRESSOS EM $\mu \mathrm{g} / \mathrm{g}$ DE AMOSTRA INTEGRAL}

\begin{tabular}{lcc|ccc}
\hline Carotenóides & \multicolumn{2}{c|}{ in natura $^{1}$} & \multicolumn{3}{c}{ Após cozimento convencional ${ }^{1}$} \\
\cline { 2 - 6 } & Média \pm DP & $\%$ & Média \pm DP & $\%$ & IV \\
\hline$\beta$-caroteno & $9,35 \pm 0,07$ & 6,35 & $12,17 \pm 3,06$ & 7,90 & $7,61-14,05$ \\
$\zeta$-caroteno & $17,79 \pm 0,16$ & 4,05 & $8,17 \pm 2,80$ & 5,30 & $3,38-11,87$ \\
Criptoflavina & $12,14 \pm 1,48$ & 7,70 & $12,65 \pm 5,67$ & 8,22 & $4,88-17,91$ \\
$\beta$-criptoxantina & $93,67 \pm 4,57$ & 5,25 & $7,99 \pm 3,99$ & 5,18 & $4,49-13,28$ \\
Anteraxantina & $79,14 \pm 48,04$ & 40,54 & $31,28 \pm 12,57$ & 20,30 & $14,78-44,97$ \\
Zeaxantina & $4,32 \pm 0,62$ & 34,24 & $79,73 \pm 13,10$ & 51,75 & $65,47-93,03$ \\
Mutatoxantina & $14,68 \pm 0,06$ & 1,87 & $2,07 \pm 1,97$ & 1,35 & $0,19-4,63$ \\
\hline Total & 231,09 & 100,00 & 154,06 & 100,00 & \\
\hline
\end{tabular}

1 = Resultados da análise de 4 a 6 amostras.

DP = Desvio Padrão; IV = Intervalo de Variação.

O teor de carotenóides encontrado na polpa in natura do piqui foi cerca de $20 \%$ inferior ao observado em estudo anterior (286,65 $\mu \mathrm{g}$ de carotenóides/g de amostra integral). Deve-se ressaltar, no entanto, que este último valor foi obtido com metodologia, cujo conteúdo total de carotenóides foi dosado com base apenas no $\beta$-caroteno (12). 


\title{
3.2 TEORES DE VITAMINA "A" DO PIQUI
}

A Tabela 2 resume os dados referentes aos carotenóides pró-vitamínicos "A" na polpa do piqui in natura e após o cozimento convencional, com teor de vitamina "A" expressos em Unidades Internacionais (UI), e Equivalentes de Retinol (ER) por $100 \mathrm{~g}$ de amostra.

\section{TABELA 2 - CAROTENÓIDES PRÓ-VITAMÍNICOS "A" E VALORES DE VITAMINA "A" DA POLPA DO PIQUI (Caryocar brasiliense Camb)}

\begin{tabular}{ccccc}
\hline Carotenóides & \multicolumn{2}{c}{ In natura } & \multicolumn{2}{c}{ Após cozimento convencional } \\
\hline & $\mathrm{UI} * / 100 \mathrm{~g}$ & ER**/100 g & UI*/100 g & ER**/100 g \\
\hline$\beta$-caroteno & 2446 & 244,7 & 2028 & 202,8 \\
Criptoflavina & 1482 & 148,2 & 1054 & 105,4 \\
$\beta$-criptoxantina & 1011 & 101,1 & 666 & 66,6 \\
\hline $\begin{array}{c}\text { Total de vitamina } \\
\text { "A" }\end{array}$ & 4939 & 494 & 3748 & 374,8 \\
\hline
\end{tabular}

* UI - Unidade Internacional ${ }^{* \star} E R$ - Equivalente de Retinol.

O total de vitamina "A" encontrado na polpa mostrou-se muito inferior ao citado na literatura, que o considera como fruto bastante rico em vitamina "A" $\pm 200.000 \mathrm{UI} / 100 \mathrm{~g}$ (10). É provável que este valor superestimado tenha sido obtido com base no total de carotenóides e não apenas dos pigmentos precursores de vitamina "A".

\subsection{EFEITO DO COZIMENTO CONVENCIONAL SOBRE OS CAROTENÓIDES PRÓ-VITAMÍNICOS "A" DA POLPA DO PIQUI}

\begin{abstract}
A Figura 1 ilustra 0 efeito do cozimento convencional sobre os carotenóides pró-vitamínicos "A" da polpa do piqui, expressando-se os valores de vitamina "A" em Equivalentes de Retinol. As perdas nos teores de vitamina "A" foram em média de $12,11 \%$. O $\beta$-caroteno apresentou perda média de $2,81 \%$, a criptoflavina de $5,72 \%$ e a $\beta$-criptoxantina de $5,13 \%$.
\end{abstract}


Considerando a perda média de $30,25 \%$ dos carotenóides, provocada pelo cozimento da polpa do piqui, verificou-se percentual de perda inferior ao encontrado na avaliação anterior. Naquela o fruto foi submetido a processo de desidratação, em estufa com circulação forçada de ar, e a degradação dos carotenóides representou cerca de $49 \%$ em relação ao total de carotenóides encontrados na polpa in natura (10). O efeito do cozimento sobre os carotenóides e o teor de vitamina "A", bem como suas perdas, foram avaliados por outros autores em diferentes hortaliças $(4,6,17)$.

\section{FIGURA 1 - EFEITO DO COZIMENTO CONVENCIONAL SOBRE OS CAROTENÓIDES PRÓ-VITAMÍNICOS "A" DA POLPA DO PIQUI (Caryocar brasiliense Camb)}

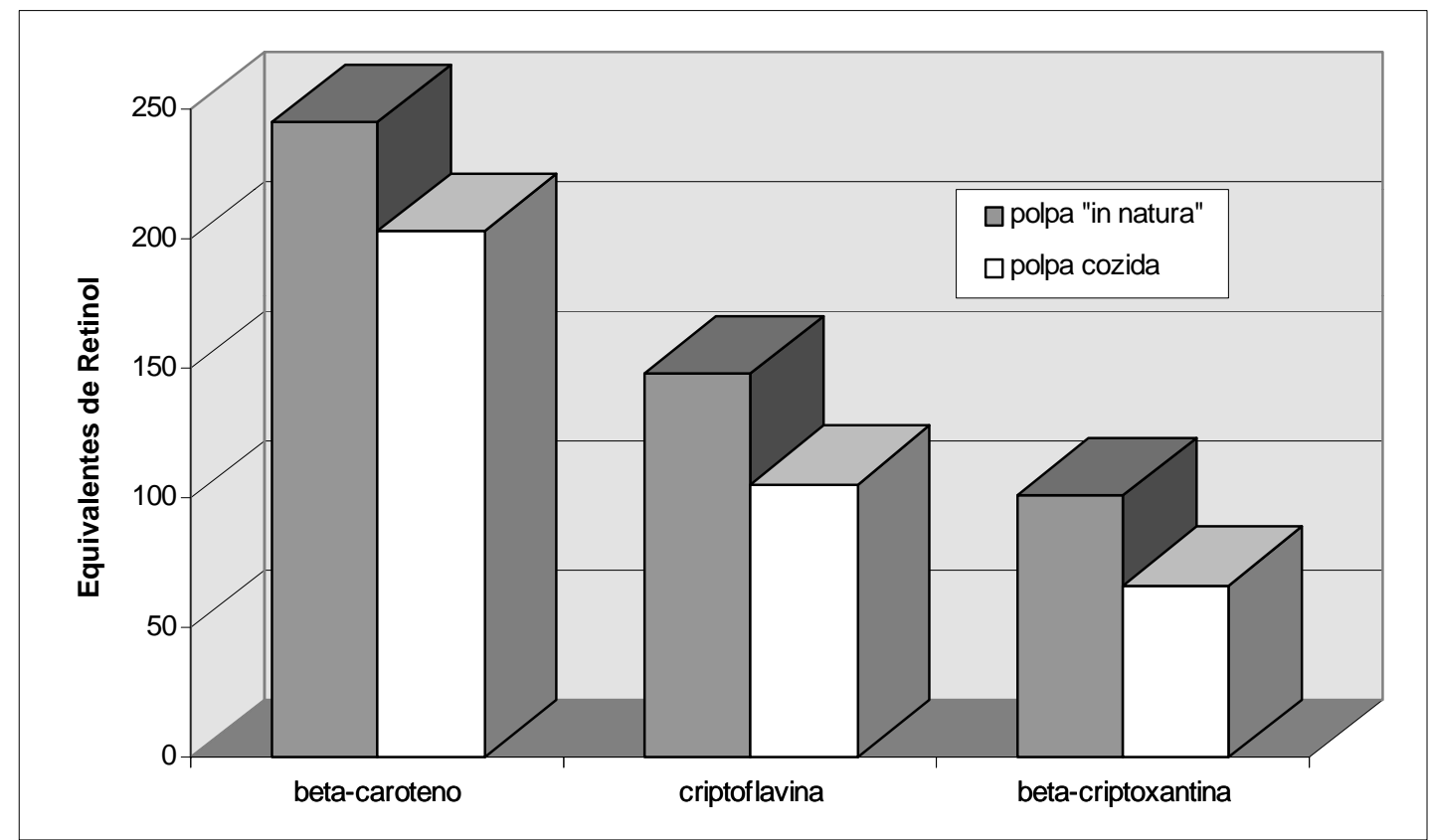

\section{CONCLUSÃO}

Os principais carotenóides encontrados na polpa do piqui (Caryocar brasiliense Camb) crua e cozida foram: $\beta$-caroteno, $\zeta$-caroteno, criptoflavina, $\beta$-criptoxantina, anteraxantina, zeaxantina e mutatoxantina.

Apresentam atividades como precursores de vitamina " $A$ ", entre os carotenóides identificados: o $\beta$-caroteno, a criptoflavina e a $\beta$-criptoxantina. $\mathrm{Em}$ todas as amostras analisadas $0 \beta$-caroteno foi o principal pigmento responsável pela atividade pró-vitamínica "A" encontrada. 
A perda de carotenóides decorrente do cozimento convencional da polpa do piqui (Caryocar brasiliense Camb) foi em média de 30,25\%, correspondendo à perda média de $12,11 \%$ no teor de vitamina "A".

\begin{abstract}
This work aimed to characterize carotenoids, to evaluate its provitamin A activity and to verify the losses of those pigments due to the conventional cooking of the pulp of piqui (Caryocar brasiliense Camb) with rice, simulating the preparation in agreement with the regional habits. Raw and cooked samples presented the same carotenoids: $\beta$-carotene, $\zeta$ carotene, cryptoflavin, $\beta$-cryptoxantin, antheraxanthin, zeaxanthin and mutatoxanthin. The average value of carotenoids was of 231,09 and $154,06 \mu \mathrm{g} / \mathrm{g}$ of raw and cooked pulp, respectively. The vitamin A content corresponded to $494 \mathrm{RE} / 100 \mathrm{~g}$ of in natura pulp and $375 \mathrm{RE} / 100 \mathrm{~g}$ of pulp after cooking. In all the samples analyzed, the $\beta$-carotene was the main responsible pigment for the provitamin $A$ activity found in the pulp. The carotenoids loss due to the conventional cooking of the pulp of piqui ( $C$. brasiliense Camb) was of $30,25 \%$, corresponding to the average loss of $12,11 \%$ in the vitamin A value.
\end{abstract}

\title{
REFERÊNCIAS
}

1 ALMEIDA, L. B.; PENTEADO, M. V. C. Ocorrência de carotenóides em raízes de cinco cultivares de mandioca (Manihot esculenta Crantz) do Estado de São Paulo. R. Farm. Bioquim. Univ. S. Paulo, São Paulo, v. 24, p. 39-49, 1988.

2 ALMEIDA, S.P. Cerrado: aproveitamento alimentar. Planaltina, DF: EMBRAPA, Centro de Pesquisa Agropecuária dos Cerrados - CPAC, 1998. $188 \mathrm{p}$.

3 BAUERNFEIND, J. C. Carotenoids as colorants and vitamin A precursors. New York: Academic Press, 1981. 938 p.

4 BIANCHINI, R.; PENTEADO, M.V.C. Carotenóides de pimentões amarelos (Capsicum annuum L.). Caracterização e verificação de mudanças com o cozimento. Ciênc. Tecnol. Aliment., v. 18, n. 3, p. 283-288, 1998.

5 BRITTON, G. Structure and properties of carotenoids in relation to function. Faseb J., Bethesda, v. 9, n. 15, p. 1551-8, 1995.

6 COSTA, M.A.L.; PENTEADO, M.V.C. Alterações decorrentes de dois tipos de cozimentos sobre os teores de carotenóides pró-vitamínicos $A$ em escarolas (Cichorium endivia L.). R. Farm. Bioquim. Univ. S. Paulo, São Paulo, v. 32, n. 2, p. 95-100,1996. 
7 DAVIES, B. H. Carotenoids. In: GOODWIN, T. W. Chemistry and biochemistry of plant pigments. New York: Academic Press, 1976. $2 \mathrm{v}$.

8 FERREIRA, M. B. Frutos comestíveis do Distrito Federal. III. Piqui, mangaba, marolo e mamãozinho. R. Cerrado (Brazil), v. 5, n. 20, p. 22-5, 1973.

9 GODOY, H.T. Mudanças na composição de carotenóides durante processamento térmico e estocagem de manga (Mangifera indica) e mamão (Carica papaya). Campinas, 1985. 111 p. Dissertação (Mestrado), Universidade Estadual de Campinas.

10 PEQUI. Guia Rural Abril, Rio de Janeiro: Abril, 1988. p. 235-236.

11 PINHEIRO-SANT'ANA, H.M.; STRINGHETA,P.C.; BRANDÃO, S.C.C.; PAEZ, H.H.; QUEIROZ, V.M.V. Evaluation of total carotenoids, $\alpha$ and B-carotene in carrots (Daucus carota L.) during home processing. Ciênc. Tecnol. Aliment., v. 18, n. 1, p. 1-148, 1998.

12 RAMOS, M.I.L. Desidratação do piqui (Caryocar brasiliense Camb): avaliação através dos teores de carotenóides totais. São Paulo, 1987. 120 p. Dissertação (Mestrado) - Faculdade de Ciências Farmacêuticas, Universidade de São Paulo.

13 ROCK, C. L.; FADA, R. D.; JACOB, R. A.; BOWEN, P. E. Update on the biological characteristics of the antioxidant micronutrients: vitamin C, vitamin E, and the carotenoids. J. Am. Diet. Assoc., Chicago, v. 96, n. 7 , p. 693-702, 1996

14 RODRIGUEZ-AMAYA, D.B. Carotenoids and food preparation: the retention of provitamin A carotenoids in prepared, processed, and stored foods. Arlington: OMNI Project, 1997. $88 \mathrm{p}$.

15 RODRIGUEZ-AMAYA, D.B. A guide to carotenoid analysis in foods. Washington: ILSI, 1999. $64 \mathrm{p}$.

16 RODRIGUEZ, D.B.; RAIMUNDO, L.C.; LEE, T.C.; SIMPSON, K.L.; CHICHESTER, C. O. Carotenoids pigments changes in ripenning Momordica charantia fruits. Ann. Bot.; London, v. 40, p. 615-24, 1976.

17 SANT'ANNA, Z.M.; PENTEADO, M. V. C. Alterações dos carotenóides decorrentes do cozimento convencional e em forno microondas de três hortaliças. R. Farm. Bioquim. Univ. S. Paulo, São Paulo, v. 32, n. 1, p. 53-8, 1996. 
18 SILVA, J. A.; SILVA, D. B.; JUNQUEIRA, N.T.V.; ANDRADE, L. R. N. Frutas nativas dos cerrados. Brasília, DF: EMBRAPA, Centro de Pesquisa Agropecuária dos Cerrados - CBAC, 1994. 166 p.

19 TRIGUEIRO, I.N.S.; PENTEADO, M.V.C. Teores de alfa e beta caroteno e atividade pró-vitamínica A de óleos de dendê brasileiros. $\mathbf{R}$. Farm. Bioquim. Univ. S. Paulo, São Paulo, v. 28, n. 1, p. 61-74, 1992.

20 YUYAMA, L. K. O.; COZZOLINO, S. M. F. Efeito da suplementação com pupunha como fonte de vitamina "A" em dieta : estudo em ratos. R. Saúde Pública, v. 30, n. 1, p. 61-6, 1996. 